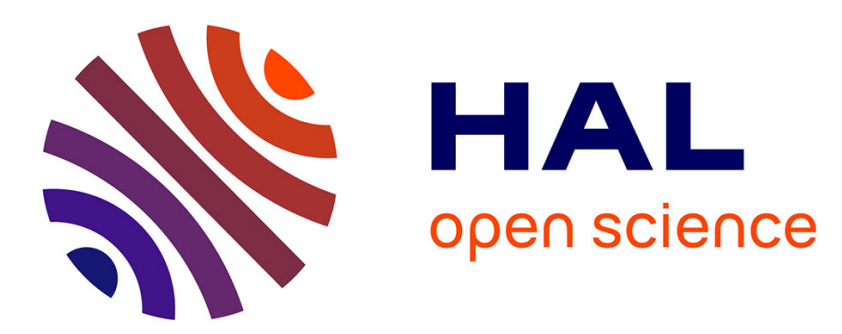

\title{
Effect of form errors on the positioning precision of over-constrained systems
}

Denis Teissandier, Yann Ledoux, Santiago Arroyave-Tobón, Vincent Delos, Jean Marc Linares

\section{- To cite this version:}

Denis Teissandier, Yann Ledoux, Santiago Arroyave-Tobón, Vincent Delos, Jean Marc Linares. Effect of form errors on the positioning precision of over-constrained systems. CIRP Annals - Manufacturing Technology, 2019, 10.1016/j.cirp.2019.04.068 . hal-02153665

\section{HAL Id: hal-02153665 https://hal.science/hal-02153665}

Submitted on 17 Jul 2019

HAL is a multi-disciplinary open access archive for the deposit and dissemination of scientific research documents, whether they are published or not. The documents may come from teaching and research institutions in France or abroad, or from public or private research centers.
L'archive ouverte pluridisciplinaire HAL, est destinée au dépôt et à la diffusion de documents scientifiques de niveau recherche, publiés ou non, émanant des établissements d'enseignement et de recherche français ou étrangers, des laboratoires publics ou privés. 


\title{
Effect of form errors on the positioning precision of over-constrained systems
}

\author{
Denis Teissandiera, Yann Ledouxa, Santiago Arroyave-Tobonc, Vincent Delos ${ }^{\mathrm{b}}$, Jean Marc Linares ${ }^{\mathrm{c}}$
}

(1)

aUniv. Bordeaux, I2M, UMR 5295, F-33400 Talence, France

${ }^{b}$ CNRS, I2M, UMR 5295, F-33400 Talence, France

${ }^{c}$ Aix Marseille Univ, CNRS, ISM, Marseille, France

This paper analyses the influence of form deviations on over-constrained mechanical systems. A mechanical clamp was equipped with LVDT sensors to measure relative displacements between its two parts to derive their relative position. Each part was measured using CMM filtering its form deviations. Part feature variations (position, orientation and dimension) and assembly clearances were aggregated on a set of $6 \mathrm{~d}$ linear constraints. From the measured local displacements another polytope is derived. These two polytopes are compared to quantify the influence of form error on the geometrical behaviour of the assembly. To do this evaluation new metrics are presented and discussed.

Precision; Positioning; Geometric modelling

\section{Introduction}

The objective of a tolerance analysis is to aggregate the geometric error variations of the constitutive parts of a mechanical system in order to check the functional requirements. Such an analysis process is based on: a model of geometric error variations, a set of relations between them derived from the topological structure of the mechanical system and numerical tools to simulate its geometrical behavior. This tolerance analysis can be done according to worst case or statistical approaches [1]. The major question is how to select the suited values of geometrical product specifications in order to control the geometric error variations (form, orientation, position and dimension) to guarantee the expected product quality. To simplify the tolerance analysis, some assumptions are usually made, among which form errors are neglected and mechanism parts are considered as infinitely rigid bodies. These assumptions could lead to inaccuracies in the computation results as shown by [2]. Only a few research works, summarized in [3], address the effect of form error variations on the geometrical behavior of an assembly. This is a key point in mechanism analysis that should be quantified. Some works are based on experimental devices to compare the simulated and the real behaviors of the assembly. However, these works were dedicated to analyze, experimentally, isostatic assemblies.

In this work, a practical case study is used to demonstrate the influence of form errors in the positioning precision on over-constrained mechanical systems. Error metrics are proposed to quantify that. In the context of Industry 4.0, these metrics are computed using a digital twin of the mechanical system. Their uses in production control are discussed in the manufacturing field. In a first part of this paper, a geometrical model for over constrained mechanisms is set up assuming no error from variations. An example of a mechanical clamp with 2 parts and their several parallel joints is treated. In the second part, based on rigid body restrictions, the influence of the form error variations on the relative position between two parts is evaluated. This evaluation is based on the comparison of the calculated and the measured displacements due to geometrical errors. To do this evaluation four new metrics are presented and discussed.

\section{Materials and methods}

The parts were modeled as infinitely rigid bodies. The displacement restrictions induced by the non-interpenetration conditions between two surfaces potentially in contact are formalized by a set of linear constraints [4, 5, 6]. Each set of contact constraints is represented by a polyhedron shown in the next section [7].

\subsection{Geometrical model for over-constrained systems}

Let us consider a contact restriction between surfaces $1, j$ (surface $j$ of part 1) and 2,j (surface $j$ of part 2), see fig. 1. The general definition of the contact restriction is given by (1). This definition is applied to a set of $N_{k}$ points (with $1 \leq k \leq k$ max and $k$ max is the number of facets for the discretization of each contact surface). At each point, the contact restriction is applied along the normal vector $\mathbf{n}_{k}$ where $d_{k}$ is the distance between $1, j$ and $2, j$. The intersection of this set of restrictions defines the polyhedron $P_{j}$.

$$
\begin{array}{r}
\mathrm{P}_{j}=\bigcap_{k}\left(\mathbf{t}_{N k_{-} \mathbf{1}, j / 2, j}\right) \cdot \mathbf{n}_{k} \leq d_{k} \Leftrightarrow \\
\mathrm{P}_{j}=\bigcap_{k}\left(\mathbf{t}_{\boldsymbol{o}_{-} \mathbf{1}, j / 2, j}+\mathbf{N}_{\mathbf{k}} \mathbf{0} \times \mathbf{r}_{1, j / 2, j}\right) \cdot \mathbf{n}_{k} \leq d_{k}
\end{array}
$$

$\mathrm{P}_{j}$ is a polyhedron of dimension 6 since each local contact restriction is a half-space $\bar{H}_{k}^{-}$in the deviation space of dimension 6 [7]. The 6 parameters $\left(R_{x}, R_{y}, R_{z}, T_{x}, T_{y}, T_{z}\right)$ are coming from the components of $\mathbf{r}_{1, j / 2, j}$ (rotation vector) and $\mathbf{t}_{0-1, j / 2,1}$ (translation vector expressed at point 0 ). The point $O$ is any point assumed to be rigidly linked to the set of points $N_{k}$. Let us consider a mechanical assembly composed of two parts: part 1 and part 2, in multiple relations by several contacts in parallel between surface $1, j$ and $2, j(1 \leq j \leq j \mathrm{max})$ : see fig. 2 . A datum $\mathrm{R}_{1}$ is associated to the surfaces $1, j$ of part 
1 by measurements. Following the same method, a datum $\mathrm{R}_{2}$ is associated to the surfaces $2, j$ of part 2 . The determination of the local distances $d_{k}$ from (1) takes into account the location deviations of $1, j$ with respect to $\mathrm{R}_{1}\left(\mathbf{d}_{1, j / \mathrm{R} 1}\right)$ and $2, j$ with respect to $R_{2}\left(d_{2, j / R 1}\right)$. The relative position between $R_{1}$ and $R_{2}$ is defined by $P_{c} . P_{c}$ is the intersection of the $n$ polyhedron $\mathrm{P}_{j}(1 \leq j \leq j \mathrm{max}$; where $j \mathrm{max}$ is the number of contact surfaces). If the contacts between parts 1 and 2 suppress the 6 degrees of freedom, $\mathrm{P}_{\mathrm{c}}$ is a polytope (i.e. a bounded polyhedron). Then the vertices of $\mathrm{P}_{\mathrm{c}}$ define the extremal values of the parameters $\left(R_{x}, R_{y}, R_{z}, T_{x}, T_{y}, T_{z}\right)$ in a deviation space. $\mathrm{P}_{\mathrm{c}}$ is the result of the intersection between (jmax $\left.\times k \max \right)$ local restrictions (i.e. half-spaces of dimension 6). Finally, we will use the general expression (2), commonly named $\mathrm{H}$ description, to define the polytope $\mathrm{P}_{c}$. According to the Minkowski-Weyl theorem [8], an equivalent definition (3) can be used for $\mathrm{P}_{c}$ where $v_{c i}$ are the vertices of $\mathrm{P}_{c}$.

$$
\begin{aligned}
\mathrm{P}_{\mathrm{c}} & =\bigcap_{j} \mathrm{P}_{j}=\bigcap_{u} \overline{\mathrm{H}}_{u}^{-} \text {with: } 1 \leq u \leq j \max \times k \max \\
\mathrm{P}_{\mathrm{c}} & =\operatorname{Conv}\left(v_{c i}\right)
\end{aligned}
$$

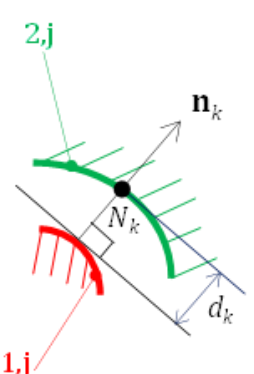

(a)

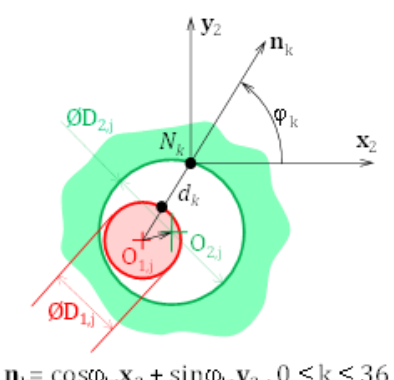

(b)

Figure 1. Local (a) and global (b) contact restrictions.

The definition (3) is commonly named the V-description of $\mathrm{P}_{c}$, where $\mathrm{P}_{\mathrm{c}}$ is defined by the convex hull of its vertices $v_{c i}$. We use this theorem in order to directly measure the relative position between parts 1 and 2 by a measured polytope $P_{m}$. A mechanical system was instrumented by sensors which measures a finite number of relative positions between parts. Each position corresponds to a set of parameters defined by a point $\operatorname{dev}_{u}$ in the deviation space. Finally, $\mathrm{P}_{\mathrm{m}}$ can be estimated by the convex hull of points $\operatorname{dev}_{u}$ according to (4) where umax is the number of measures to generate the points $\operatorname{dev} u$.

$$
\mathrm{P}_{m}=\operatorname{Conv}\left(\operatorname{dev}_{u}\right) \quad 1 \leq u \leq u \max
$$

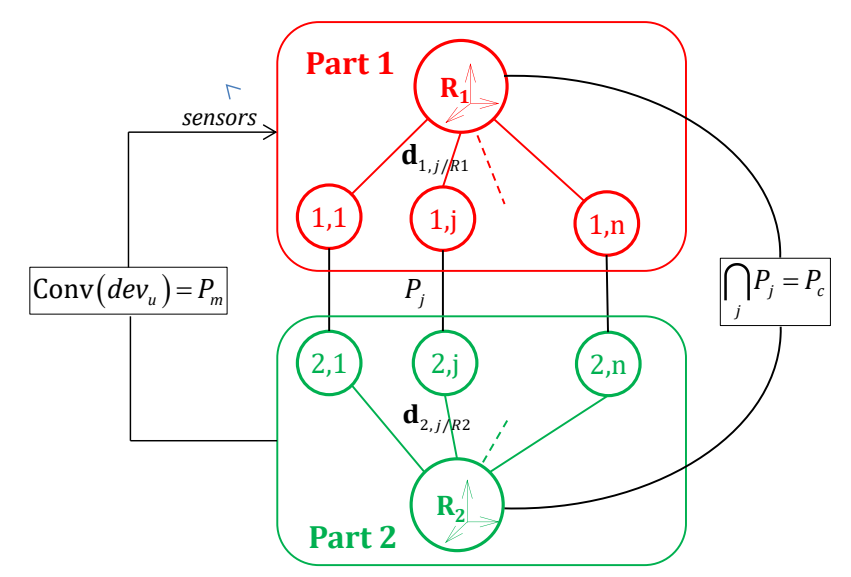

Figure 2. Contact restrictions at the assembly level.

\subsection{Experimental protocol}

Our aim is to evaluate the influence of the form error variations on the relative position between two parts in contact in the case of an over constrained assembly. This evaluation is based on the comparison between the calculated polytope $\mathrm{P}_{\mathrm{c}}$ and the measured polytope $\mathrm{P}_{\mathrm{m}}$. The surfaces $1, j$ and $2, j$ are modeled by substitute surfaces through the measurement process, in order to eliminate the form deviations by least square best-fit method (i.e. gaussian filters). So, the polytope $\mathrm{P}_{\mathrm{c}}$ is the aggregation of orientation, position and dimension variations. The convex hull of points $\left(\operatorname{dev}_{u}\right)$ derives from direct measurements of the relative position between parts. The polytope $\mathrm{P}_{\mathrm{m}}$ is then the aggregation of all the possible sources of geometrical variations of surfaces $1, j$ and $2, j$ (i.e. orientation, position, dimension and then form errors).

The study is carried out on a mechanical system inspired by a clamp (fig. 3a and b). The constitutive parts 1 and 2 are in multiple contacts by three couples of pin $1, j$ - hole $2, j(1 \leq j \leq 3)$ with a nominal diameter equal to $\emptyset 10$. These couples are evenly distributed on a nominal circle (Ø80) (fig. 3c). Two additional datums 1,4 and 2,4 respectively on part 1 and part 2, are nominally located on the center of this circle. These respective center holes $\mathrm{O}_{1,4}$ and $\mathrm{O}_{2,4}$ with a fixed square on 
part 1 are used to measure the relative position between datum $R_{1}$ and datum $R_{2}$ respectively linked to parts 1 and 2 . We define contact restrictions assuming the contact zones between pins and holes lie in the same plane (i.e. the common plane between parts). As a consequence, the relative position between $\mathrm{R}_{1}$ and $\mathrm{R}_{2}$ is defined by a small rotation along a normal to the common plane and two orthogonal translations in this plane. The polytopes $P_{c}$ and $P_{m}$ are both $3 d$ polytopes. The origin of datum $\mathrm{R}_{1}$ is defined by $O_{1,1}$ and one axis by the square. The origin of datum $\mathrm{R}_{2}$ is defined by $\mathrm{O}_{2,1}$ and one axis by a line $\left(\mathrm{O}_{2,1}, \mathrm{O}_{2,4}\right)$.

(a)
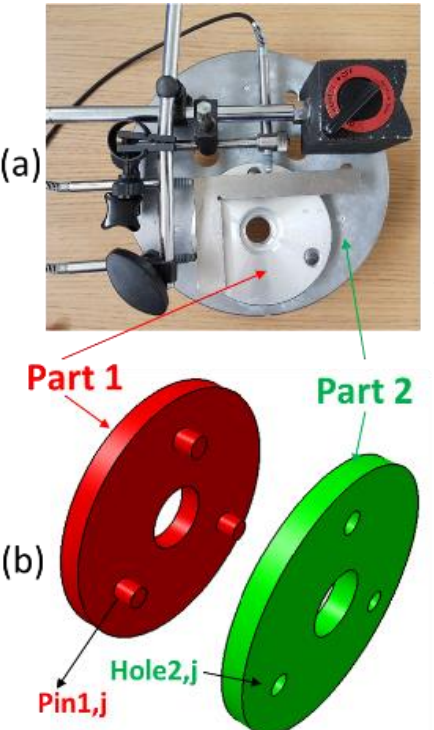

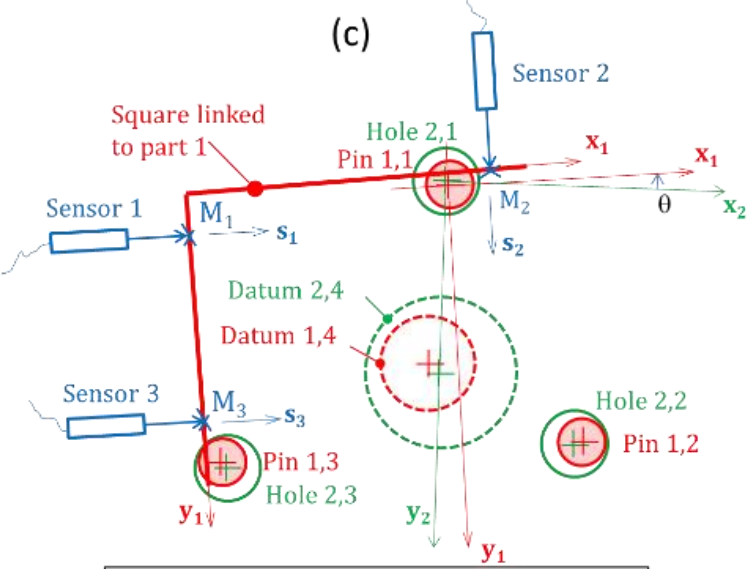

$0_{1, j}:$ center of pin $1, j \quad 1 \leq j \leq 3$

$0_{2, j}$ : center of hole $2, \mathrm{j} 1 \leq \mathrm{j} \leq 3$

Expression of polytopes at point $\mathrm{O}=\mathrm{O}_{2,4}$

Figure 3. Experimental device (a); CAD part (b); geometric setting of the clamp (c).

The five steps of the analysis protocol are depicted in fig. 4.

First, part 1 is measured to estimate the location of surfaces $1, j$ in $\mathrm{R}_{1}$ and their diameters $\emptyset D_{1, j}$ (step 1). By analogy, surfaces $2, j$ in $\mathrm{R}_{2}$ and their diameters $\emptyset D_{2, j}$ are estimated on the part 2 . Each surface is measured by 8 points evenly distributed with a CMM machine (Renishaw ${ }^{\circledR}$ TP20, measurement uncertainty: $7 \mu \mathrm{m}$ in volume). Although they are not used in the computation of polytope $\mathrm{P}_{c}$, the form deviations of each contact surface are also evaluated. In step 2, part 1 is assembled on part 2 and fixed in an arbitrary location such as their common planes are in contact. Then, in step 3 , the square and the hole 1,4 are measured in $\mathrm{R}_{2}$ and the LVDT sensors are initialized in this configuration. This is a major step in the best-fit of the origins of polytopes of $\mathrm{P}_{\mathrm{c}}$ and $\mathrm{P}_{\mathrm{m}}$. In step 4, an operator manually moved part 1 with respect to part 2 reaching as many extremal positions as possible. The motions between parts are measured by the three LVDT sensors (DP5 probes of Solartron Metrology® with accuracy of $0.5 \mu \mathrm{m}$ ). Finally, all the data are saved to be used for the computation of polytopes $\mathrm{P}_{\mathrm{c}}$ and $\mathrm{P}_{\mathrm{m}}$ (step 5).

\subsection{Computed polytope $\mathrm{P}_{\mathrm{c}}$}

The contact restrictions between pin 1,j and hole 2,j derive from definition (1), and $\mathrm{P}_{j}$ can be expressed as (5).

$$
\begin{aligned}
& \mathbf{P}_{\boldsymbol{j}}=\bigcap_{\boldsymbol{k}}\left(\mathbf{t}_{\boldsymbol{o}_{-} \mathbf{1}, \mathbf{j} / \mathbf{2}, \boldsymbol{j}}+\mathbf{N}_{\mathbf{k}} \mathbf{0} \times \mathbf{r}_{\mathbf{1}, \mathbf{j} / \mathbf{2}, \mathbf{j}}\right) \cdot \mathbf{n}_{\boldsymbol{k}} \leq d_{k} \\
& \text { with } d_{k}=\left(\frac{D_{2, j}-D_{1, j}}{2}\right)+\mathbf{0}_{1, j} \mathbf{o}_{2, j} \cdot \mathbf{n}_{k}
\end{aligned}
$$

The couples $N_{k}, \mathbf{n}_{k}$ are deduced from a mesh built on the CAD model of the clamp. This couple and the point $\mathrm{O}$ control the normal of each half-space $\bar{H}_{k}^{-}$. An operand $\mathrm{P}_{j}$ is generated with $k \mathrm{max}=36$ such that the maximal deviation between the mesh and the surfaces of pin $1, j$ and hole $2, j$ is less than $1 \mu \mathrm{m}$ [7]. The other parameters to compute a local distance $d_{k}$ are coming from the diameters and the location deviations of pin $1, j$ and hole $2, j$. Their values derive from the measurements performed in steps 1 and 2 of the synoptic (see fig. 4). Least square filtering is used to remove the form deviations to others for pins $1, j$ and holes $2, j$. From (2) the polytope $\mathrm{P}_{\mathrm{c}}$ can be computed as shown in fig. 5. 


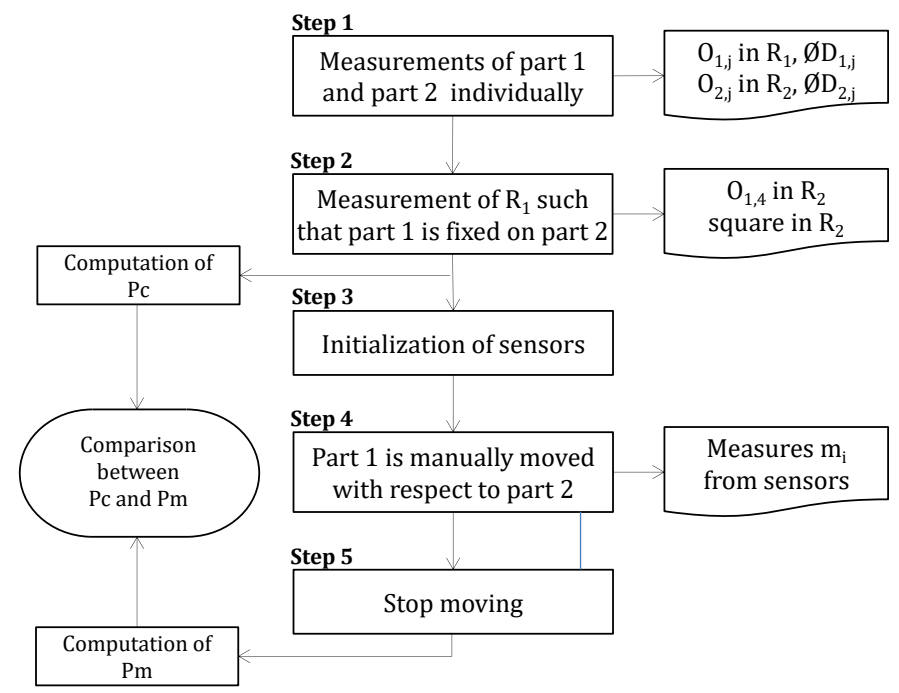

Figure 4. Experimental protocol.

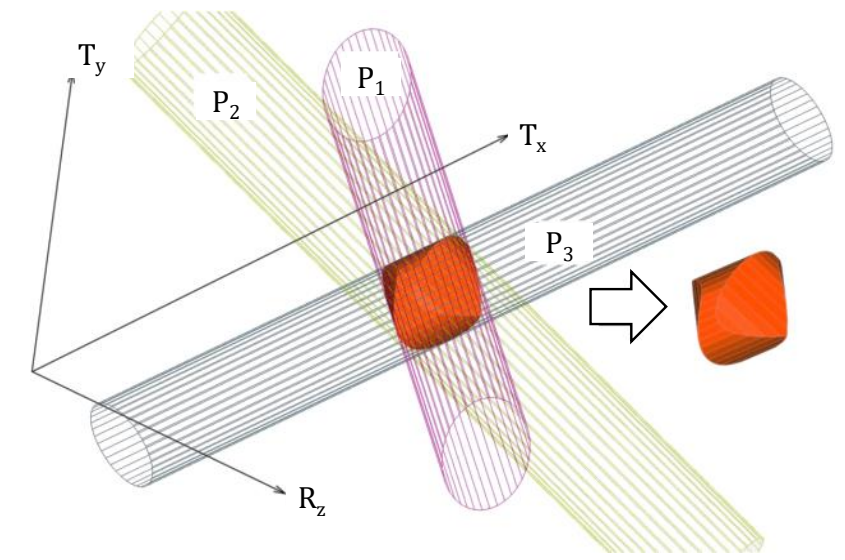

Figure 5. Polytope $P_{c}=P_{1} \cap P_{2} \cap P_{3}$.

\subsection{Measured polytope $\mathrm{P}_{\mathrm{m}}$}

From local measures of the sensors, we obtain a set of relations using equation (6). These relations can be expressed at a common point 0 assumed to be rigidly linked with the points $\mathrm{M}_{\mathrm{i}}$. Then the parameters $R_{z}, T_{x}$ and $T_{y}$ can be determined from (6) with (7) and (8) by (9).

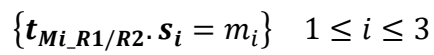

$$
\begin{aligned}
& t_{M i_{-} R 1 / R 2}=t_{O_{\_} R 1 / R 2}+r_{R 1 / R 2} \times M_{i} O \\
& \text { (7) } \\
& \boldsymbol{s}_{\boldsymbol{i}}=\left(s_{i x}, s_{i y}, 0\right) \quad \boldsymbol{O} \boldsymbol{M}_{\boldsymbol{i}}=\left(O M_{i x}, O M_{i y}, 0\right)
\end{aligned}
$$

Finally, from $u$ max records of triplets $m_{i}$ we can deduce a cloud of points $\operatorname{dev}_{u}$ in a deviation space $\left(R_{z}, T_{x}, T_{y}\right)$. 

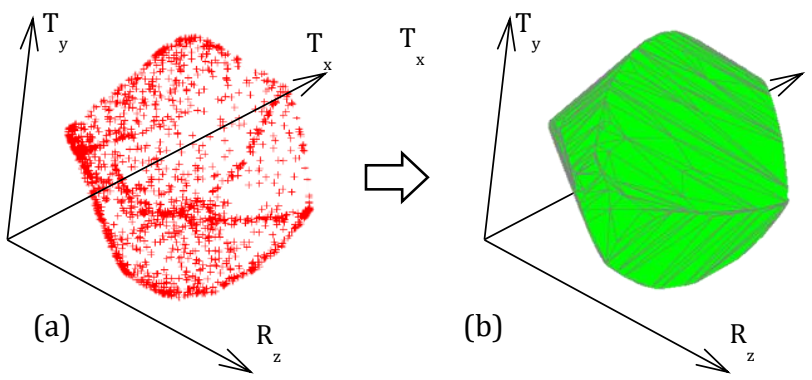

Figure 6. Cloud of 2000 points (a) and its convex hull $P_{m}(b)$.

\section{Results and discussions}

\subsection{Measured polytope $\mathrm{P}_{\mathrm{m}}$}

Fig. 6a gives an example with 2000 records of triplets $m_{i}$. Fig $6 \mathrm{~b}$ shows the polytope $\mathrm{P}_{\mathrm{m}}$ resulting from the convex hull of 2000 points. The vertices of $P_{m}$ are the extremal displacements between parts 1 and 2 reached by the manual operation. The point density on the boundary of $\mathrm{P}_{\mathrm{m}}$ is very heterogeneous.

\subsection{Computed polytope $\mathrm{P}_{\mathrm{c}}$}

The results of the CMM measurement of parts 1 and 2 are displayed in table 1 . The vertices of $\mathrm{P}_{c}$ result from the intersections of its half-spaces initially generated on the nodes of a regular mesh, see fig. 5 and 7.
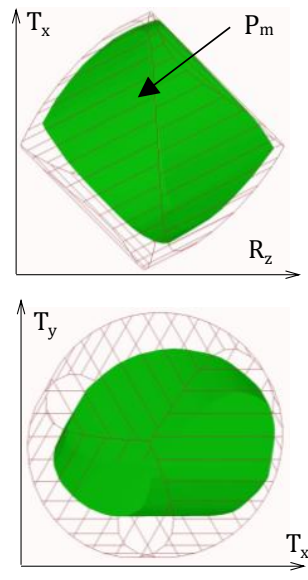
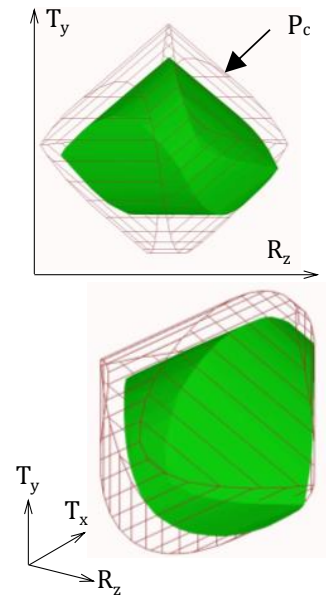

Figure 7. Projections of polytopes $\mathrm{P}_{c}$ and $\mathrm{P}_{\mathrm{m}}$ in canonical views.

\subsection{Comparison between polytopes $\mathrm{P}_{\mathrm{m}}$ and $\mathrm{P}_{\mathrm{c}}$}

The difference between the shape of polytopes $P_{c}$ and $P_{m}$ is due to the form deviations. Based on equation (5), the distance $d_{\mathrm{k}^{*}}$ between a pin $1, j$ and a hole $2, j$ is redefined by (10).

$$
d_{k *}=d_{k}+f v_{k}
$$

The parameter $f v_{\mathrm{k}}$ is the accumulation of form error deviation of a pin $1, j$ with respect to a hole $2, j$ at a node $N_{\mathrm{k}}$ along the normal $\mathbf{n}_{\mathrm{k}}$, see fig. 1 . It was filtered by the best-fit process after the CMM measurement of parts 1 and 2 and it is not taken into account in (5). In consequence, form error variations generate a translation $f v_{\mathrm{k}}$ of the half-space $\bar{H}_{k}^{-}$along its normal. In general, the parameters $f v_{\mathrm{k}}$ have an influence on the boundary of the intersection of these half-spaces. Each vertex of a polytope defines an extremal position between the parts 1 and 2 and is created by a minimum of 3 concurrent hyperplanes in dimension 3 . These hyperplanes are the boundaries of the half-spaces deriving from some points $\mathrm{N}_{\mathrm{k}}$ which define the contact zone between the parts 1 and 2 . The relation between the half-spaces and the vertices ensures the complete traceability between nodes $N_{k}, \mathbf{n}_{k}$ and the extremal positions extracted from its faces [7]. The comparison between polytopes $\mathrm{P}_{\mathrm{m}}$ and $\mathrm{P}_{\mathrm{c}}$ cannot be done based on properties of their lattices. Therefore, the direct estimation of the values $f v_{\mathrm{k}}$ is not possible. In response to this, new geometrical metrics are proposed to compare $\mathrm{P}_{\mathrm{m}}$ and $\mathrm{P}_{\mathrm{c}}$ such as the bounding boxes, the mass centers, the volumes and the Hausdorff distances [9]. The bounding box of $\mathrm{Pm}_{\mathrm{m}}$ is included inside the bounding box of $\mathrm{P}_{c}$. According to the parameters $\left(R_{z}, T_{x}, T_{y}\right)$, the ratios of the three extends of the bounding boxes are $88 \%, 87 \%$ and $67 \%$, see table 2 . If we want to limit the global motions of one part compare to the other one, this bounding 
box computation is a useful quantification. It is also, a well indication of the positioning precision of the parts (small volume meaning a small motion).

Table 1. Measurement results of individual parts 1 and 2 in $\mathrm{R}_{2}$ in $\mathrm{mm}$

\begin{tabular}{|c|c|c|c|c|c|c|}
\hline Surface & 1,1 & 1,2 & 1,3 & 2,1 & 2,2 & 2,3 \\
\hline Form dev & 0.014 & 0.011 & 0.012 & 0.007 & 0.009 & 0.007 \\
\hline Diameter & 10.004 & 10.008 & 10.006 & 10.554 & 10.558 & 10.557 \\
\hline Loc $\mathrm{x}$ & -0.273 & 34.904 & -34.422 & 0.000 & 34.663 & -34.619 \\
Loc y & 39.973 & 20.070 & 20.056 & 40.000 & 19.995 & 19.995 \\
\hline
\end{tabular}

Table 2. Bounding boxes of polytopes

\begin{tabular}{|l|c|c|c|}
\hline Bounding box & $R_{z}\left(10^{-3} \mathrm{rad}\right)$ & $T_{x}(\mathrm{~mm})$ & $T_{y}(\mathrm{~mm})$ \\
\hline $\mathrm{P}_{\mathrm{c}}$ & {$[-7.321 ; 6,384]$} & {$[-0.510 ; 0.023]$} & {$[-0.302 ; 0.207]$} \\
\hline $\mathrm{P}_{\mathrm{m}}$ & {$[-6.190 ; 5.860]$} & {$[-0.456 ; 0.007]$} & {$[-0.217 ; 0.133]$} \\
\hline Ratio $\mathrm{P}_{\mathrm{m}} / \mathrm{P}_{\mathrm{c}}$ & $88 \%$ & $87 \%$ & $67 \%$ \\
\hline
\end{tabular}

These results do not integrate the correlations between the parameters $\left(R_{z}, T_{x}, T_{y}\right)$.

Table 3. Mass centres of polytopes

\begin{tabular}{|l|c|c|c|}
\hline Mass center & $R_{z}\left(10^{-3} \mathrm{rad}\right)$ & $T_{x}(\mathrm{~mm})$ & $T_{y}(\mathrm{~mm})$ \\
\hline $\mathrm{G}_{\mathrm{c}}\left(\mathrm{P}_{\mathrm{c}}\right)$ & -0.468 & -0.247 & -0.056 \\
\hline $\mathrm{G}_{\mathrm{m}}\left(\mathrm{P}_{\mathrm{m}}\right)$ & -0.165 & -0.211 & -0.065 \\
\hline $\mathbf{G}_{\mathrm{c}} \mathbf{G}_{\mathrm{m}}$ & 0.303 & 0.026 & -0.009 \\
\hline
\end{tabular}

Assuming that the relative position of parts is randomly distributed in the polytope, the vector GcGm then gives the average of the relative location between datum $\mathrm{R}_{1}$ and $\mathrm{R}_{2}$ in terms of $\left(R_{z}, T_{x}, T_{y}\right)$ in the $3 \mathrm{~d}$ deviation space, see table 2 . The magnitude and the direction of this vector are strongly correlated to the distribution of $f v_{\mathrm{k}}$ on the contact surfaces.

Table 4. Volumes of polytopes in $10^{-3} \mathrm{~mm}^{2} \cdot \mathrm{rad}$

\begin{tabular}{|c|c|c|c|}
\hline & $\mathrm{P}_{\mathrm{c}}$ & $\mathrm{P}_{\mathrm{m}}$ & $\mathrm{P}_{\mathrm{c}} \cap \mathrm{P}_{\mathrm{m}}$ \\
\hline Volume & 46.04 & 25.58 & 25.46 \\
\hline
\end{tabular}

Furthermore, the polytope $P_{m}$ is not included inside the polytope $P_{c}$ but the volume of $P_{c} \cap P_{m}$ is very closed to the volume of $\mathrm{P}_{\mathrm{m}}$, see table 4. These volumes indicate the inclusion rate and traduce if the polytope $\mathrm{P}_{\mathrm{c}}$ increase or not the extremal displacements of $\mathrm{P}_{\mathrm{m}}$. These two-last metrics $\left(\mathbf{G}_{\mathrm{c}} \mathbf{G}_{\mathrm{m}}\right.$ and volumes) can help a designer to validate a design choice in a preliminary design stage.

Finally, the deviations from the tessellated boundary of $\mathrm{P}_{\mathrm{m}}$ with respect to the boundary of $\mathrm{P}_{\mathrm{c}}$ can be deduced by a set of distances, see fig. 8. Each distance is the Hausdorff distance between a point from the boundary of $P_{m}$ and $P_{c}$. Each signed distance indicates if the form deviation must be added or subtracted along a given direction in the neighborhood of an extremal position between the parts 1 and 2 . These distances and their directions can lead to detect what half-space

$\bar{H}_{k}^{-}$are on the boundary of $\mathrm{Pm}$. Thus, it is possible to investigate about the evolution of the contact zones with and without form error variations in a geometrical model. This diagnosis can lead to detect precisely where are the influent zones on the relative position between parts 1 and 2 . Furthermore, this diagnosis can be useful to analyze the load transfer between the parts in order to predict the mechanical behavior of the assembly. These new metrics were integrated in the open source software (i2m.u-bordeaux.fr/politopix).

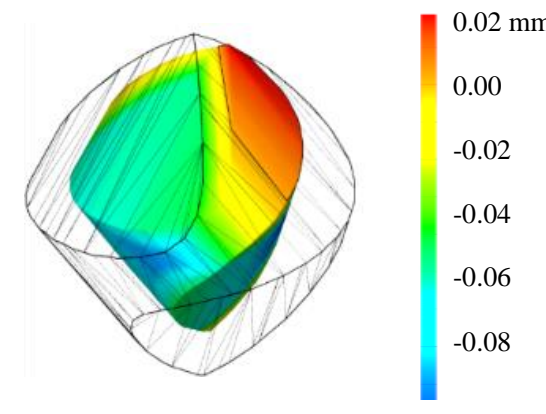

Figure 8. Hausdorff distances between tessellation of $\mathrm{P}_{m}$ with respect to the boundary of $\mathrm{P}_{c}$.

\section{Conclusion}

A complete analysis protocol to evaluate the influence of the form error variations on the relative position between parts of an over constrained assembly was presented. This study proposes new metrics to detect and localize assembly interferences induced by form deviations. Moreover, it brings to the fore the influence of form errors in the positioning precision. The four proposed metrics were: Bounding boxes, Mass centers, Volumes of polytopes and Hausdorff distances. 
In future, the metric uncertainties will be evaluated in order to take into account the effect of assumptions (rigid body, small screw displacement, surface discretization,...) and measure uncertainties. These metrics can help designers to take decisions in geometrical product specification. Additionally, they could be used in Industry 4.0, for example in smart part pairing, for choosing the constitutive parts that warrantee an assembly with no interference. This could be done, in real time in the production chain, by digital twin assembly simulation. The knowledge of the assembly interference localization opens the way to automatic processes to repair high added value parts.

\section{References}

[1] Cao, Y., Liu, T.,Yang, J., 2018, A comprehensive review of tolerance analysis models, Int J Adv Manuf Technol, 97/5-8: 3055-3085.

[2] Grandjean, J., Ledoux, Y., Samper S., 2013, On the role of form defects in assemblies subject to local deformations and mechanical loads, Int J Adv Manuf Technol, 65/9:1769-1778.

[3] Xingyu, Y., Alex, B., 2019, Review and Comparison of Form Error Simulation Methods for Computer-Aided Tolerancing, ASME J Comput Inf Sci Eng, 19/010802:1-16.

[4] Fleming, A., 1988, Geometric relationships between toleranced features, Artif. Intell. 37:403-412.

[5] Giordano, D. Duret, S. Tichadou, R. Arrieux., 1992, Clearance space in volumic dimensioning, CIRP Ann. - Manuf. Technol. 41/1:565-568.

[6] Davidson, J., Mujezinovic, A., Shah, J., 2002, A new mathematical model for geometric tolerances as applied to round faces, ASME J. Mech. Des. 124:609622.

[7] Arroyave Tobon, S., Teissandier, D., Delos, V., 2017, Tolerance analysis with polytopes in HV-description, ASME J Comput Inf Sci Eng, 17/041011:1-9.

[8] Ziegler, G. M. (2012). Lectures on polytopes (Vol. 152). Springer Science \& Business Media.

[9] Ushakov, V.N., Lebedev, P.D., Tarasyev, A.M., Ushakov, A.V., 2015, Optimization of the Hausdorff distance between convex polyhedrons in R3, IFAC, 48/25:197-201. 